\title{
Crystal structure of 1,6-di(ferrocenyl)-3,4-dihydroxy-hexa-2,4-diene- 1,6-dion, $\mathrm{Fe}_{2}\left(\mathrm{C}_{5} \mathrm{H}_{5}\right)_{2}\left(\mathrm{C}_{16} \mathrm{H}_{12} \mathrm{O}_{4}\right)$
}

\author{
P. Mayer ${ }^{1}$, O. E. Woisetschläger ${ }^{\mathrm{II}}$ and W. Beck ${ }^{*, \mathrm{I}}$ \\ I Ludwig-Maximilians-University, Department of Chemistry, Butenandtstrasse 5-13 (D), D-81377 Munich, Germany \\ I Dr. O. K. Wack Chemie GmbH, Forschung \& Entwicklung, Bunsenstr. 6, D-85053 Ingolstadt, Germany
}

Received January 18, 2002, accepted and available on-line March 22, 2002; CCDC-No. $1267 / 786$

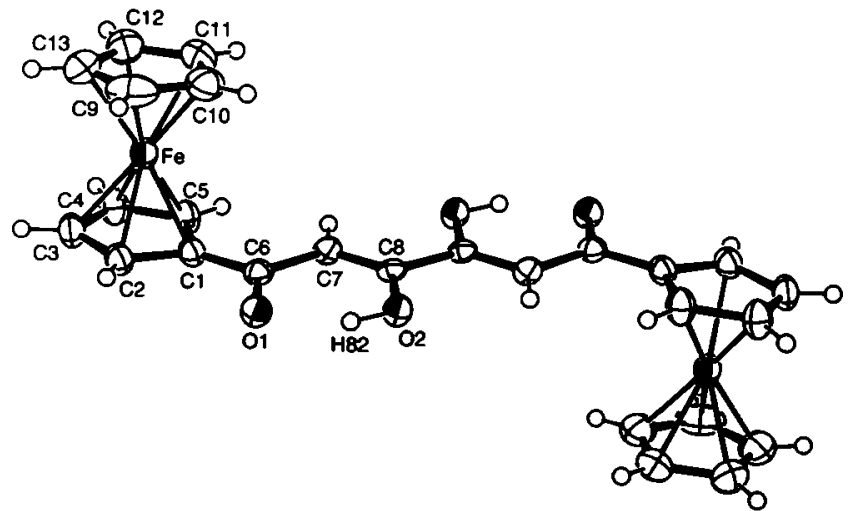

Abstract

$\mathrm{C}_{26} \mathrm{H}_{22} \mathrm{Fe}_{2} \mathrm{O}_{4}$, monoclinic, $P 12_{1} / n 1$ (No. 14), $a=5.9905(2) \AA$, $b=15.0253(4) \AA, c=12.0497(4) \AA, \beta=90.945(1)^{\circ}, V=1084.4 \AA^{3}$, $Z=2, R_{\mathrm{gt}}(F)=0.038, w R_{\text {ref }}\left(F^{2}\right)=0.080, T=200 \mathrm{~K}$

\section{Source of material}

The title complex was synthesized from acetylferrocene, potassium hydride and diethyloxalate in thf, as described in [1]. Violet plates were obtained from a concentrated solution of the title compound in dichloromethane.

\section{Discussion}

The title molecule $\mathrm{Fe}_{2}\left(\mathrm{C}_{5} \mathrm{H}_{5}\right)_{2}\left(\mathrm{C}_{16} \mathrm{H}_{12} \mathrm{O}_{4}\right) 1$ possesses an inversion centre (figure). The bond lengths and bond angles clearly show that the title complex has also in the solid state the keto enol structure and that the two hydroxy groups are in 3,4 positions of the hexadiene-dion rather than in 1,6. Important bond lengths and bond angles are: $d(\mathrm{Cl}-\mathrm{C} 6)=1.458(3) \AA, d(\mathrm{C} 6-\mathrm{O} 1)=1.258(3) \AA$, $d(\mathrm{C} 6-\mathrm{C} 7)=1.447(3) \AA, d(\mathrm{C} 7-\mathrm{C} 8)=1.346(3) \AA, d(\mathrm{C} 8-\mathrm{O} 2)=$ $1.335(3) \AA, d\left(\mathrm{C} 8-\mathrm{C} 8^{\prime}\right)=1.474(3) \AA, \angle \mathrm{O} 1-\mathrm{C} 6-\mathrm{C} 7=120.6(2)^{\circ}$, $\angle \mathrm{C} 6-\mathrm{C} 7-\mathrm{C} 8=120.5(2)^{\circ}, \angle \mathrm{C} 7-\mathrm{C} 8-\mathrm{O} 2=123.2(2)^{\circ}$, $\angle C 7-C 8-\mathrm{C}^{\prime}=123.7(2)^{\circ}$. The bond lengths in 1 are very similar to those in a bis(palladium) complex of the dianion of 1 $-\left(1-2 \mathrm{H}^{+}\right)\left[\mathrm{Pd}(\mathrm{Cl}) \mathrm{P}\left(\mathrm{C}_{6} \mathrm{H}_{4} \mathrm{CH}_{3}\right)_{3}\right]_{2}[2]-$ with the exception that in that complex the four $\mathrm{C}-\mathrm{O}$ distances are almost identical $(1.282(4) \AA, 1.279(4) \AA$ ), as expected. In the structure of 1 , there is no symmetrical hydrogen bridge between the carbonyl groups. The bond length of $\mathrm{O} 2-\mathrm{H} 82$ is $0.88(3) \AA$ while the distance between $\mathrm{H} 82$ and the acceptor $\mathrm{Ol}$ is $1.70(3) \AA$. The bond angle $\angle \mathrm{O} 2-\mathrm{H} 82-\mathrm{O} 1$ is $155(3)^{\circ}$. The angles between the least-square plane of $\mathrm{O} 1, \mathrm{O} 2, \mathrm{C} 6, \mathrm{C} 7$, and $\mathrm{C} 8$ and the $\mathrm{C}_{5} \mathrm{H}_{5}$ units are $9.58(13)^{\circ}$ and $10.22(17) \AA$, respectively, indicating slight deviation from coplanarity. A stronger deviation is found in methyl ferrocenemonocarboxylate [3] for the ester and $\mathrm{C}_{5} \mathrm{H}_{5}$ units $\left(13.07(18)^{\circ}, 11.52(17)^{\circ}\right)$.

Table 1. Data collection and handling.

\begin{tabular}{ll}
\hline Crystal: & $\begin{array}{l}\text { black-violet prism, } \\
\text { size } 0.02 \times 0.03 \times 0.25 \mathrm{~mm}\end{array}$ \\
Wavelength: & Mo $K_{\alpha}$ radiation $(0.71069 \AA)$ \\
$\mu:$ & $13.69 \mathrm{~cm}^{-1}$ \\
Diffractometer, scan mode: & Kappa CCD, $\varphi$ \\
$2 \theta_{\max }:$ & $54.94^{\circ}$ \\
$N(h k l)_{\text {measured, }} N(h k l)_{\text {unique: }}:$ & 18698,2479 \\
Criterion for $I_{\text {obs, }} N(h k l)_{\mathrm{gt}}:$ & $I_{\text {obs }}>2 \sigma\left(I_{\mathrm{obs}}\right), 1837$ \\
$N(\text { param })_{\text {refined: }}$ & 189 \\
Programs: & SIR97 [4], SHELXL-97[5], PLATON [6], \\
& ORTEP-III [7]
\end{tabular}

Table 2. Atomic coordinates and displacement parameters (in $\AA^{2}$ ).

\begin{tabular}{llllll}
\hline Atom & Site & $x$ & $y$ & $z$ & $U_{\text {iso }}$ \\
\hline H(82) & $4 e$ & $-0.359(5)$ & $0.056(2)$ & $0.954(3)$ & $0.06(1)$ \\
H(2) & $4 e$ & $-0.375(5)$ & $0.288(2)$ & $0.719(2)$ & $0.036(7)$ \\
$H(3)$ & $4 e$ & $-0.149(4)$ & $0.371(2)$ & $0.586(2)$ & $0.034(7)$ \\
$H(4)$ & $4 e$ & $0.234(5)$ & $0.305(2)$ & $0.588(3)$ & $0.047(8)$ \\
$H(5)$ & $4 e$ & $0.233(5)$ & $0.181(2)$ & $0.726(2)$ & $0.044(8)$ \\
H(7) & $4 e$ & $0.133(4)$ & $0.093(2)$ & $0.878(2)$ & $0.024(6)$ \\
H(9) & $4 e$ & $-0.211(6)$ & $0.426(2)$ & $0.919(3)$ & $0.08(1)$ \\
H(10) & $4 e$ & $0.028(5)$ & $0.310(2)$ & $1.004(3)$ & $0.06(1)$ \\
H(11) & $4 e$ & $0.389(5)$ & $0.314(2)$ & $0.921(3)$ & $0.06(1)$ \\
H(12) & $4 e$ & $0.393(6)$ & $0.439(2)$ & $0.782(3)$ & $0.08(1)$ \\
H(13) & $4 e$ & $0.007(6)$ & $0.503(3)$ & $0.780(3)$ & $0.09(1)$ \\
& & & & & \\
\hline
\end{tabular}


Table 3. Atomic coordinates and displacement parameters (in $\AA^{2}$ ).

\begin{tabular}{|c|c|c|c|c|c|c|c|c|c|c|}
\hline Atom & Site & $x$ & $y$ & $z$ & $U_{11}$ & $U_{22}$ & $U_{33}$ & $U_{12}$ & $U_{13}$ & $U_{23}$ \\
\hline $\mathrm{Fe}$ & $4 e$ & $0.03557(6)$ & $0.32821(2)$ & $0.78506(3)$ & $0.0275(2)$ & $0.0309(2)$ & $0.0346(2)$ & $-0.0006(2)$ & $-0.0016(1)$ & $0.0077(2)$ \\
\hline$O(1)$ & $4 e$ & $-0.3779(3)$ & $0.1399(1)$ & $0.8588(2)$ & $0.0217(8)$ & $0.0336(9)$ & $0.040(1)$ & $0.0014(7)$ & $0.0011(7)$ & $0.0050(8)$ \\
\hline$O(2)$ & $4 e$ & $-0.2891(2)$ & $0.0208(1)$ & $1.0006(2)$ & $0.0170(8)$ & $0.0317(9)$ & $0.039(1)$ & $0.0012(7)$ & $0.0032(7)$ & $0.0084(8)$ \\
\hline$C(1)$ & $4 e$ & $-0.0999(4)$ & $0.2067(2)$ & $0.7510(2)$ & $0.027(1)$ & $0.030(1)$ & $0.028(1)$ & $0.002(1)$ & $-0.002(1)$ & $0.003(1)$ \\
\hline$C(2)$ & $4 e$ & $-0.2341(4)$ & $0.2755(2)$ & $0.7017(2)$ & $0.030(1)$ & $0.034(1)$ & $0.032(2)$ & $0.003(1)$ & $-0.003(1)$ & $0.005(1)$ \\
\hline$C(3)$ & $4 e$ & $-0.1000(4)$ & $0.3232(2)$ & $0.6277(2)$ & $0.039(1)$ & $0.035(1)$ & $0.033(2)$ & $0.003(1)$ & $-0.003(1)$ & $0.011(1)$ \\
\hline$C(5)$ & $4 e$ & $0.1201(4)$ & $0.2138(2)$ & $0.7052(2)$ & $0.032(1)$ & $0.037(1)$ & $0.036(2)$ & $0.007(1)$ & $0.004(1)$ & $0.008(1)$ \\
\hline$C(6)$ & $4 e$ & $-0.1740(4)$ & $0.1449(2)$ & $0.8362(2)$ & $0.028(1)$ & $0.025(1)$ & $0.027(1)$ & $-0.001(1)$ & $-0.001(1)$ & $-0.003(1)$ \\
\hline$C(7)$ & $4 e$ & $-0.0120(4)$ & $0.0890(2)$ & $0.8930(2)$ & $0.018(1)$ & $0.026(1)$ & $0.033(1)$ & $0.0005(9)$ & $0.003(1)$ & $0.002(1)$ \\
\hline$C(8)$ & $4 e$ & $-0.0772(3)$ & $0.0302(1)$ & $0.9702(2)$ & $0.017(1)$ & $0.021(1)$ & $0.031(1)$ & $-0.0011(8)$ & $0.0003(9)$ & $-0.0044(9)$ \\
\hline$C(9)$ & $4 e$ & $-0.0731(6)$ & $0.4142(2)$ & $0.9045(3)$ & $0.042(2)$ & $0.064(2)$ & $0.067(2)$ & $-0.004(2)$ & $0.001(2)$ & $-0.031(2)$ \\
\hline$C(10)$ & $4 e$ & $0.0630(6)$ & $0.3477(2)$ & $0.9529(3)$ & $0.064(2)$ & $0.055(2)$ & $0.036(2)$ & $-0.019(2)$ & $-0.004(2)$ & $0.001(2)$ \\
\hline$C(11)$ & $4 e$ & $0.2738(5)$ & $0.3523(2)$ & $0.9055(3)$ & $0.043(2)$ & $0.054(2)$ & $0.056(2)$ & $-0.006(2)$ & $-0.017(2)$ & $0.007(2)$ \\
\hline$C(12)$ & $4 e$ & $0.2708(6)$ & $0.4214(2)$ & $0.8268(3)$ & $0.046(2)$ & $0.050(2)$ & $0.061(2)$ & $-0.014(2)$ & $-0.007(2)$ & $0.009(2)$ \\
\hline
\end{tabular}

\section{References}

1. Woisetschläger, O. E.; Geisbauer, A.; Polborn, K.; Sünkel, K.; Beck, W.: Spacer verbrickte Bis-, Tris- und Tetrakis(ferrocenyl)-1,3-Diketone. Z. Anorg. Allg. Chem. 625 (1999) 2164-2168.

2. Woisetschläger, O. E.; Geisbauer, A.; Polborn, K.; Beck, W.: Koordinationschemie von bis(ferrocenyl)substituierten 1,3-Diketonaten mit Ruthenium, Rhodium, Iridium und Palladium. Z. Anorg. Allg. Chem. 626 (2000) 766-774.

3. Beck, W.; Woisetschläger, O. E.; Mayer, P.: Crystal structure of methyl ferrocenemonocarboxylate. Z. Kristallogr. NCS 216 (2001) 403-404

4. Altomare, A.; Burla, M. C.; Camalli, M.; Cascarano, G. L.; Giacovazzo, C.; Guagliardi, A.; Moliterni, A. G. G.; Polidori, G.; Spagna, R.: SIR97: a new tool for crystal structure determination and refinement. J. Appl. Crystallogr. 32 (1999) 115-119.
5. Sheldrick, G. M.: SHELXL-97. Program for the Refinement of Crystal Structures. University of Göttingen, Germany 1997.

6. Spek, A. L.: PLATON, A Multipurpose Crystallographic Tool, Utrecht University, Utrecht, The Netherlands 1999. Windows version used (Farrugia, L. J., University of Glasgow).

7. Bumett, M. N.; Johnson, C. K.: ORTEP-III: Oak Ridge Thermal Ellipsoid Plot Program for Crystal Structure Illustrations, Oak Ridge National Laboratory Report ORNL-6895, USA 1996. Windows version used (Farrugia, L. J., University of Glasgow). 